\title{
Effect of familiarity and category contrast on stimulus and response priming
}

\author{
SCOTT A. WEISGERBER and PEDER J. JOHNSON \\ University of New Mexico, Albuquerque, New Mexico
}

\begin{abstract}
In a letter-symbol classification task, flanking incompatible symbols slowed response latencies to letter targets, whereas incompatible letter flankers did not slow the classification of symbol targets. The conditions surrounding this asymmetry in response competition were investigated in five experiments. The results showed that: (1) the asymmetry was not related to the familiarity of the symbol targets or to the prime-target interval; (2) when the classification involved familiar and unfamiliar symbols, the asymmetry remained (i.e., there was less interference associated with the unfamiliar symbol targets), but there was now significant response competition associated with both symbol categories; and (3) with a mixed-category task (i.e., letters and symbols assigned to both responses), the symbol targets continued to be less interfered with by both letter and symbol incompatible flankers. These findings were interpreted as suggesting that response competition can be influenced by both classification decision rules and cohesiveness of exemplars comprising a category.
\end{abstract}

In recent years, a number of researchers have investigated the effects of visual contextual information on speeded classification performance (e.g., Eriksen \& Schultz, 1979; Flowers \& Wilcox, 1982; Grice \& Gwynne, 1985). These studies have demonstrated that contextual stimuli, even when irrelevant to the task at hand, may influence both perceptual and response components of the classification task. These two different loci of context effects were isolated by applying the subtractive procedure to the classification task as it was developed by Eriksen and Eriksen (1974) and Taylor (1977). In this task, subjects classify target stimuli that are spatially flanked by contextual stimuli, which are of one of three types in their relation to the target stimulus: (1) identical-primes that are physically identical to the target; (2) compatible-primes that are not identical to the target, yet are associated with the same response as that with which the target is associated; and (3) incompatible-primes that are associated with a different response than the response with which the target is associated. The perceptual component (referred to as stimulus priming) is estimated by comparing the difference in response latencies between the identical and compatible prime conditions, whereas the response component (referred to as response priming) is estimated by comparing the compatible and incompatible prime conditions.

The magnitude of the stimulus-priming effect appears to be greatest when the stimulus onset asynchrony (SOA) between the prime and the target is approximately

The authors wish to thank Lucinio Santos-Gomez and Nancy Nelson for their helpful assistance in data collection and analysis. We also wish to thank an anonymous reviewer for suggesting Experiment 5 . Requests for reprints should be sent to Peder J. Johnson, Department of Psychology, University of New Mexico, Albuquerque, NM 87131.
200-300 msec. As the SOA approaches zero, stimuluspriming effects tend to disappear (Grice \& Gwynne, 1985; Taylor, 1977). It appears that at least part of this stimuluspriming effect involves an encoding type of process (Grice, Boroughs, \& Canham, 1984; Kirsner \& Dunn, 1985) that is often interpreted in terms of a logogen type of model (Morton, 1969, 1979). Specifically, the prime preactivates permanent memory codes, which speeds the subsequent encoding of the identical target stimulus.

The magnitude of the response-priming effect is greatest when the prime and target are presented simultaneously, and it decreases as the SOA between the prime and the target increases out to 200 or $300 \mathrm{msec}$ (Flowers \& Wilcox, 1982; Taylor, 1977). The inclusion of a neutral prime condition as a baseline has shown that an incompatible prime produces interference, whereas a compatible prime may produce a small amount of facilitation if it precedes the target by at least $50 \mathrm{msec}$ (Flowers \& Wilcox, 1982; Grice \& Gwynne, 1985). Thus, it appears that the response-priming effect includes both a facilitory and an inhibitory component. The inhibitory component, assumed to be the result of incorrect response tendencies produced by the incompatible prime, is referred to by Eriksen and Schultz (1979) as response competition. The facilitory component is usually assumed to be a result of correct response tendencies produced by a compatible prime, but there is some evidence that the response-priming effect may also involve a stimulus-evaluation component (Coles, Gratton, Bashore, Eriksen, \& Donchin, 1985).

Much of the evidence for stimulus and response priming has been based on studies in which familiar English letters were used as stimuli. An exception to this is an experiment by Shapiro and Krueger (1983), in which subjects classified uppercase English letters as either normal or distorted (i.e., normal uppercase letters with one line 
segment either added or deleted). When the targets were normal, Shapiro and Krueger found both stimulus- and response-priming effects, but in the case of the distorted targets, neither a stimulus- nor a response-priming effect was evident. The absence of stimulus priming with the distorted targets was seen by Shapiro and Krueger as consistent with the predictions coming from a logogen type of model (Morton, 1969, 1979), in that the distorted letters did not have a well-established representation in permanent memory. Their response-priming results indicated that a normal letter prime failed to interfere with the speeded classification of a distorted target, whereas a distorted letter prime slowed response latencies to a normal letter. This asymmetry in response priming poses a problem for the notion of response competition. If response competition simply reflects the influence of conflicting response tendencies associated with the prime, then both familiar and unfamiliar primes should produce response competition.

Our primary aim in the present study was to investigate the boundary conditions surrounding the occurrence of response priming. Specifically, are the unexpectedly asymmetrical response-priming effects reported by Shapiro and Krueger (1983) reliable, and what conditions are necessary to produce them? Secondarily, we were interested in determining whether it was possible to observe a stimulus-priming effect with unfamiliar stimuli.

\section{EXPERIMENT 1}

In Experiment 1, subjects classified four English letters and four foreign letters, which were drawn from the Arabic and Hebrew alphabets, into opposing categories. These two types of targets were flanked by identical, compatible, or incompatible primes, allowing us to look at the effects of familiarity on both stimulus and response priming. Because it is well established that stimulus and response priming are highly sensitive to time-course effects (Flowers \& Wilcox, 1982; Grice \& Gwynne, 1985; Taylor, 1977), and because it is not known how stimulus familiarity may influence this time course, it was necessary to investigate a range of prime-target intervals. Toward this end, three separate groups of subjects were observed, under 0-, 100-, and 300-msec SOA primetarget intervals.

In the present experiments, we were primarily interested in studying the nonstrategic effects of primes on processing (cf. Posner \& Snyder, 1975). With this in mind, the subjects were given instructions that encouraged them to consciously ignore the primes. In addition, the relative frequency of the three different types of primes was set at a level that made them nonpredictive of the target stimulus or the correct response (Miller, 1987; Taylor, 1977). To achieve zero stimulus-information value in a two-choice classification task that involves eight different stimuli (four in each category), an identical prime should occur on oneeighth (.125) of the trials. Similarly, to achieve zero response-information value, the prime should predict the correct response on half of the trials. Therefore, zero stimulus- and response-information value is achieved in an eight-trial block comprising one identical prime, three compatible primes, and four incompatible primes.

\section{Method}

Subjects. Fifty-four students from introductory psychology courses at the University of New Mexico participated in the experiment for extra credit toward their grade. Eighteen subjects were arbitrarily assigned to each of the three prime-target SOA conditions. The data from 1 additional subject were discarded, because the subject had prior knowledge of the unfamiliar stimuli. Each subject participated individually in a single experimental session that lasted approximately $1 \mathrm{~h}$.

Apparatus and Stimuli. The presentation of stimuli, control of timing intervals, and recording of data were all carried out on a TERAK 8510 microcomputer. The familiar stimuli were the English letters F, H, S, and R. The unfamiliar stimuli consisted of three Hebrew letters and one Arabic letter, all four of which can be seen in Figure 1. The characters, presented on a TERAK monitor, were white, appearing on a dark background. When viewed from a distance of $60 \mathrm{~cm}$, each character subtended $.34^{\circ}$ of visual angle horizontally and $.43^{\circ}$ vertically. The stimuli were presented in the center of a $25 \times 30 \mathrm{~mm}$ outline of a rectangle, which served as a fixation field. The prime stimuli were presented on either side of the target stimulus, separated by one blank character space. This resulted in an edge-to-edge separation of $.34^{\circ}$ of visual angle. The entire stimulus array of the target and two primes subtended $1.72^{\circ}$ of visual angle horizontally and $.43^{\circ}$ vertically. The subjects responded to the stimuli by pressing one of two specially marked keys located on the right and left sides of the bottom row of the keyboard.

Procedure. The fixation field remained on the screen throughout the experimental session. Each trial began with the presentation of a tone that served as the warning signal. The tone preceded the onset of the target stimulus by $1,000 \mathrm{msec}$ in each of the three SOA groups. The prime-target SOA interval was measured from the onset of the prime to the onset of the target. Both the primes and the target remained on the screen until they were terminated by the response. If a response was not made within $2 \mathrm{sec}$ following the presentation of the target, the stimuli were terminated and the trial was scored as an error. Following a 2.5 -sec intertrial interval, the next warning signal was presented. Feedback was presented on the viewing screen for $1 \mathrm{sec}$ immediately following the subject's response. The message "CORRECT" was displayed on correct trials, and the message "ERROR" was displayed on incorrect trials.

The subjects were instructed that they were to respond with respect to whether the target stimulus was a letter or a symbol (i.e., an unfamiliar letter). They were asked to respond as rapidly and as accurately as possible. Half of the subjects used the left key to respond to the letters and the right key to respond to the symbols. The other half of the subjects received the opposite response mapping. The subjects were also told that the task was a test of distractibility, and that they should try to ignore the prime characters presented on both sides of the target.

An experimental session consisted of 480 experimental trials ( 240 letter trials and 240 symbol trials) and 96 practice trials. The percentages of identical, compatible, and incompatible trials were 12.5 ,

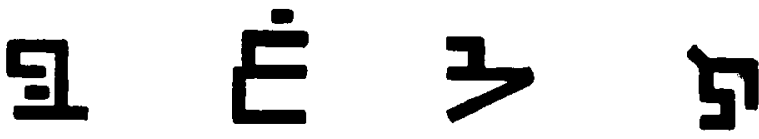

Figure 1. Unfamiliar symbol stimuli. 
37.5 , and 50 , respectively. The sequence of trial types was randomized within 16 trial blocks.

\section{Results and Discussion}

Response priming. The means of subjects' median reaction times (RTs) and the mean percentages of errors are shown in Table 1. The analysis of the responsepriming effect indicated that there was a highly significant response-priming $\times$ familiarity interaction $[F(1,51)=$ $181.7, p<.001]$. This interaction reflected the fact that there was a significant response-priming effect for the letter targets $[F(1,51)=286.8, p<.001]$, but that there was no evidence of a response-priming effect for the unfamiliar targets $(F<1.0)$.

Response priming also interacted with SOA $[F(2,51)=6.35, p<.01]$. Examination of Table 1 indicates that the interaction resulted from the responsepriming effect's being larger in the 100-msec SOA group than in the 0- or 300-msec SOA groups. Simple analyses indicated that the response-competition effect was significant for letter targets in each of the three SOA groups [all $F \mathrm{~s}(1,17)>44.0, p \mathrm{~s}<.001$ ], and nonsignificant for the unfamiliar symbol targets [all $F_{\mathrm{s}}(1,17)<3.0$, ps $>$.10]. There was no evidence of an SOA $\times$ familiarity interaction $[F(2,51)<1.0]$, nor of a three-way interaction $[F(2,51)=1.27, p>.10]$.

The analysis of error data yielded a similar pattern of findings. The interaction of response priming $\times$ familiarity was significant $[F(1,51)=33.2, p<.001]$, indicating that for letter targets there were more errors on incompatible trials than on compatible trials $[F(1,51)=$ $40.9, p<.001]$, whereas for symbol targets there was no difference between compatible and incompatible trials $[F(1,51)=1.28, p>.10]$. There was also a responsepriming $\times$ SOA interaction $[F(1,51)=6.1, p<.01]$, again indicating that the difference between compatible and incompatible trials was larger in the 100 -msec SOA group (see Table 1).

Table 1

Mean Reaction Times (RT) and Proportions of Errors (E) for Experiment 1 as a Function of Stimulus Onset Asynchrony (SOA), Target Familiarity, and Type of Prime

\begin{tabular}{|c|c|c|c|c|}
\hline \multirow[b]{3}{*}{ Type of Prime } & \multicolumn{4}{|c|}{ Target } \\
\hline & \multicolumn{2}{|c|}{ Familiar } & \multicolumn{2}{|c|}{ Unfamiliar } \\
\hline & RT & $E$ & RT & $E$ \\
\hline \multicolumn{5}{|c|}{0 -msec SOA } \\
\hline Identical & 503 & 0.2 & 548 & 0.7 \\
\hline Compatible & 512 & 0.2 & 560 & 0.7 \\
\hline Incompatible & 569 & 2.3 & 568 & 0.9 \\
\hline \multicolumn{5}{|c|}{ 100-msec SOA } \\
\hline Identical & 460 & 0.6 & 480 & 0.9 \\
\hline Compatible & 471 & 0.7 & 520 & 1.3 \\
\hline Incompatible & 549 & 5.4 & 530 & 2.3 \\
\hline \multicolumn{5}{|c|}{300 -msec SOA } \\
\hline Identical & 412 & 0.9 & 431 & 2.4 \\
\hline Compatible & 441 & 1.2 & 484 & 1.8 \\
\hline Incompatible & 485 & 2.9 & 475 & 1.3 \\
\hline
\end{tabular}

These results are consistent with the asymmetrical response-priming effects reported by Shapiro and Krueger (1983). They reported a response-priming effect for normal letters and none for distorted letters. Because this asymmetry in response priming was present for all three of the prime-target SOA intervals investigated, it appears that it is not time-course dependent.

Stimulus priming. The analysis of the stimulus-priming effect indicated that there was a significant prime type $\times$ familiarity $\times$ SOA interaction $[F(2,51)=3.53, p<.05]$. As can be seen from Table 1, there was a general increase in the stimulus-priming effect with increases in the SOA, and this effect was more pronounced for the unfamiliar symbol targets than for the letter targets. There was a significant stimulus-priming $\times$ familiarity interaction in both the 100 - and the 300 -msec SOA groups $[F \mathrm{~s}(1,17)=$ 17.2 and 17.3 , respectively, $p s<.01]$. The stimuluspriming $\times$ familiarity interaction was nonsignificant in the 0 -msec SOA group $(F<1)$. Analysis of the stimuluspriming effect for the letter targets in each SOA group indicated that there was a significant stimulus-priming effect in the 300 -msec SOA group $[F(1,17)=56.0$, $p>.001$, but the effect was only marginally significant in the 100 - and 0 -msec SOA groups $[F(1,17)=3.6$ and 4.0 , respectively, $p s<.1]$. For the unfamiliar symbols, the stimulus-priming effect was significant in both the 100 and the 300-msec SOA groups $[F(1,17)=29.7$ and 118.1, respectively, both $p s<.001$ ], but in the 0-msec SOA group the effect was only marginal $[F(1,17)=3.96, p<$ .1]. Analysis of error data indicated that the error rates did not differ for the identical and compatible prime conditions for the familiar or unfamiliar symbols across the different SOAs (all $F_{\mathrm{s}}<1.05$ ).

These findings indicate that a stimulus-priming effect was present for both familiar and unfamiliar target stimuli and that the effect decreased at the shorter prime-target intervals. The observation that stimulus priming decreased with the shorter prime-target intervals is a common finding in this literature (Grice et al., 1984; Posner \& Snyder, 1975). The demonstration of a stimulus-priming effect for the unfamiliar targets in the 100- and 300-msec SOA groups contrasts with Shapiro and Krueger's (1983) finding that distorted letter stimuli did not produce a stimuluspriming effect.

In Experiment 1, there appeared to be smaller responsepriming and larger stimulus-priming effects for the symbol targets than for the letter targets. These prime-target interactions may, however, be questioned on the grounds that the compatible prime condition was used as the baseline in estimating both stimulus and response priming. It is possible that these interactions reflect the differential influence of compatible primes on letter and symbol targets. In Experiment 2, we attempted to rectify this problem by including a neutral baseline control.

\section{EXPERIMENT 2}

In Experiment 1, the compatible prime condition served as the baseline for assessing the relative magnitude of 
stimulus and response priming. To use the compatible prime as the baseline control presents a problem in how the familiarity effects observed in Experiment 1 should be interpreted. This uncertainty arises from the fact that compatible primes have been shown to facilitate the processing of the target. This facilitation occurs via both response biasing (see Grice \& Gwynne, 1985) and categorical priming (i.e., the facilitation effect is larger when the prime and the target are both members of the same semantic category; see Flowers \& Wilcox, 1982; Shaffer \& LaBerge, 1979). If the facilitation from compatible primes is less for the unfamiliar symbols than it is for the letters, then there should be a corresponding underestimation of the response-priming effect and an overestimation of the stimulus-priming effect. Another possibility is that the compatible primes produce equivalent amounts of facilitation for both letters and symbols and that the absence of a significant response-priming effect for the symbol targets resulted from incompatible primes' producing less response competition when the target was an unfamiliar symbol.

In Experiment 2, a neutral baseline condition was added to provide a more analytical assessment of the loci of priming effects. A neutral condition usually involves the presentation of extraneous flankers or primes (i.e., stimuli that are not used as targets; see Jonides \& Mack, 1984). This type of neutral condition would have been inappropriate in the present classification task, because any potentially neutral stimulus might be perceived as being relatively more familiar or more unfamiliar and thereby create a response bias. For this reason, we used a no-noise neutral condition similar to that used by Grice and Gwynne (1985, Experiment 2). In the no-noise neutral condition, the target was presented alone with no flankers or primes.

As Jonides and Mack (1984) have discussed, there may be no truly valid neutral condition. Grice and Gwynne (1985) suggested that their no-noise neutral condition might be a "fast" neutral, relative to the more conventional neutral condition in which the target is flanked by irrelevant characters. Despite these problems, the inclusion of the neutral condition in the present Experiment 2 should help to clarify the extent to which the difference in response priming between the familiar and the unfamiliar stimuli was based on response facilitation or response competition.

\section{Method}

The method used in Experiment 2 was identical to that used in Experiment 1, except for the following changes: Each trial started with an auditory warning signal and the presentation of a plus sign $(+)$ that served as a fixation point. The target stimulus was presented immediately above the fixation point, and the prime stimuli were presented on either side of the target as in Experiment 1. The SOA between the primes and the target was $100 \mathrm{msec}$. The fixation point remained on the screen until the prime stimuli were presented (or would have been presented in the no-noise condition). One-third of the trials were neutral, and primes were not presented on these trials. On trials in which primes were presented, the stimulus validity remained at a chance level $(12.5 \%)$. Eighteen additional students from the pool used in Experiment 1 served as subjects.

\section{Results and Discussion}

Response priming. The means of subjects' median RTs and the mean percentages of errors are presented in Table 2. First, a comparison between the compatible and neutral conditions showed that RTs in the compatible conditions were approximately $10 \mathrm{msec}$ faster for both the familiar and the unfamiliar targets. This main effect was marginally significant $[F(1,17)=3.4, p<.1]$, but more important, there was no indication that category facilitation interacted with familiarity $[F(1,17)<1]$. These same analyses conducted on error data showed no significant effects (all $F \mathrm{~s}<1$ ). The analyses indicate that category facilitation is unrelated to familiarity.

Having shown that the letter and symbol targets were equally facilitated by compatible primes, we next compared performance in the neutral conditions with that in the incompatible prime condition. The analysis of RTs showed a significant effect for the letters $[F(1,17)=$ $50.5, p<.001]$, but none for the unfamiliar symbols $[F(1,17)<1]$, resultihg in a significant interaction with familiarity $[F(1,17)=80.1, p<.001]$. The analysis of error rates showed the same pattern as did the RT analysis, with more errors on incompatible trials than on neutral trials $[F(1,17)=26.3, p<.001]$, and a significant interaction with familiarity $[F(1,17)=20.6, p<.001]$. The interaction reflected the fact that for letters there were more errors on incompatible trials than on neutral trials $[F(1,17)=27.8, p<.001]$, whereas there was no difference in the error rates for the unfamiliar symbols $[F(1,17)<1]$.

The results indicate that the difference in response priming between the familiar and unfamiliar stimuli is entirely the result of response competition. This conclusion is consistent with the results from previous studies with neutral trials, in which it has been shown that most of the difference in mean RTs between compatible and incompatible conditions is related to response competition (e.g., Grice \& Gwynne, 1985). It would appear that in the present study, either the letter primes are not a source of response competition in classifying the unfamiliar targets or the symbol targets are not susceptible to response competition.

Stimulus priming. The test of stimulus priming involved a comparison between identical and neutral trials. There was a significant main effect of stimulus priming $[F(1,17)=40.7, p<.001]$, indicating that RTs were

Table 2

Mean Reaction Times (RT)

and Proportions of Errors (E) for Experiment 2, as a Function of Target Familiarity and Type of Prime

\begin{tabular}{lccccc}
\hline & \multicolumn{4}{c}{ Target } \\
\cline { 2 - 3 } \cline { 5 - 6 } \multicolumn{1}{c}{ Type of Prime } & \multicolumn{2}{c}{ Familiar } & & Unfamiliar \\
\cline { 2 - 3 } \cline { 5 - 6 } & RT & E & & RT & E \\
\hline Identical & 459 & 1.4 & & 483 & 0.8 \\
Response-compatible & 477 & 1.6 & & 511 & 2.7 \\
Response-incompatible & 543 & 6.6 & & 522 & 2.7 \\
Neutral & 486 & 1.3 & & 522 & 2.5 \\
\hline
\end{tabular}


faster on identical trials than on neutral trials. The interaction of stimulus priming and familiarity was not significant $[F(1,17)=1.98, p>.1]$, which indicated that there was not a significant difference in the magnitude of the stimulus-priming effect for the letters relative to the symbols. The analysis of the error data indicated that the main effect of stimulus priming was significant $[F(1,17)=10.1, p<.05]$, as was the interaction of stimulus priming and familiarity $[F(1,17)=13.6$, $p<.05]$. This interaction reflected the fact that for the familiar symbols there was not a significant difference in the error rates on identical and neutral trials $[F(1,17)<1]$, whereas for the unfamiliar symbols there were more errors on neutral trials than on identical trials $[F(1,17)=17.1, p<.001]$. Assuming a speedaccuracy tradeoff, this interaction may reflect an underestimation of the magnitude of the stimulus-priming effect for the unfamiliar symbols.

In summary, when a neutral condition was used as the baseline to estimate stimulus priming, the results showed that familiar and unfamiliar stimuli produced equivalent magnitudes of stimulus priming. This finding serves to question the assumption that stimulus priming requires the existence of a well-established memory representation. Of course it remains possible that a memory code for the unfamiliar stimuli was acquired early in the course of the experiment.

\section{EXPERIMENT 3}

The results from Experiment 2 indicated that the difference in response priming between the letter and symbol targets was related to response competition. Specifically, the incompatible letter primes did not interfere with the speeded classification of the symbol target. Either the letter primes were not a source of competition or the unfamiliar category was not subject to the influence of response competition. Neither of these possibilities is consistent with the idea of response competition. Furthermore, it has been shown repeatedly that an incompatible letter prime produces reliable response-competition effects (e.g., Eriksen \& Schultz, 1979; Grice \& Gwynne, 1985; Taylor, 1977). The absence of response competition in Experiments 1 and 2 was unexpected on both theoretical and empirical grounds. In an attempt to gain some understanding of the boundary conditions related to the occurrence of response competition, we began investigating characteristics of the letter-symbol task that distinguish it from classification tasks in which symmetrical priming effects are normally found.

An obvious difference between the symbol category used in Experiments 1 and 2 and the letter categories used in prior work is the unfamiliarity of the exemplars. In Experiment 3 , we investigated the possibility that the unfamiliarity of the Hebrew and Arabic characters constituting the symbol category was responsible for the absence of response competition. The four Hebrew and Arabic characters were replaced with four familiar symbols (\#, ?, 3, and $\uparrow$ ). These four familiar symbols were contrasted with the same four English letters that constituted the letter category in the previous experiments. Thus, the only change in Experiment 3 involved the familiarity of the characters in the symbol category.

\section{Method}

The method used in Experiment 3 was identical to that used in Experiment 2, except that there were no neutral trials and the unfamiliar stimuli were replaced by four familiar, heterogeneous symbols. The category of familiar symbols consisted of a pound sign. (\#), a question mark (?), an upward pointing arrow ( 1 ), and the number three (3). The SOA between the primes and the target was $100 \mathrm{msec}$. Eighteen additional students from the population described previously served as subjects.

\section{Results}

Response priming. The means of subjects' median RTs and the mean proportions of errors are shown in Table 3. As in the previous experiments, there was a highly significant response priming $\times$ category interaction $[F(1,17)=99.8, p<.001]$. This interaction resulted from the response-priming effect's being significant for the letters $[F(1,17)=82.5, p<.001]$ and nonsignificant for the familiar symbols $[F(1,17)<1]$. The anal$y$ sis of the error data also showed a significant responsepriming $\times$ category interaction $[F(1,17)=5.12, p<$ $.05]$. Analysis of simple effects showed that there were more errors on incompatible trials than on compatible trials for both the letters $[F(1,17)=18.2, p<.001]$ and the familiar symbols $[F(1,17)=7.0, p<.05]$.

These findings indicate that the asymmetry in response priming is not specific to the unfamiliar nature of the stimuli constituting the symbol category. Apparently some other property of the symbol categories underlies the asymmetry effect.

Stimulus priming. There was a significant stimuluspriming $\times$ category interaction $[F(1,17)=9.39, p<$ $.001]$, reflecting a larger effect for the familiar symbols than for the letters. Simple analyses indicated that the stimulus-priming effect was significant for both the letters $[F(1,17)=11.1, p<.01\}$ and the familiar symbols $[F(1,17)=24.5, p<.001]$. The analysis of the errors indicated that the identical and compatible trials did not

Table 3

Mean Reaction Times (RT) and Proportions of Errors (E) for Experiment 3, as a Function of Target Category and Type of Prime

\begin{tabular}{lccccc}
\hline & \multicolumn{4}{c}{ Target } \\
\cline { 2 - 3 } \multicolumn{1}{c}{ Type of Prime } & \multicolumn{2}{c}{ Letter } & & \multicolumn{2}{c}{ Familiar Symbol } \\
\cline { 2 - 3 } \cline { 5 - 6 } \multicolumn{1}{c}{ RT } & & E & & RT & E \\
\hline Identical & 467 & 0.9 & & 491 & 1.7 \\
Response-compatible & 480 & 0.7 & & 522 & 1.2 \\
Response-incompatible & 534 & 3.5 & & 524 & 2.6 \\
\hline
\end{tabular}


differ for either the letters or the familiar symbols (both $F$ s $<1$ ), and there was no evidence of an interaction $(F<1)$.

\section{EXPERIMENT 4}

On the basis of the results from Experiment 3, it appears that the response-priming asymmetry effect is not related to specific properties of the stimuli constituting the symbol category. There remain two other general properties related to the letter-symbol classification task that could be responsible for the asymmetry effect. One pertains to the structure of the symbol category itself. In contrast to the letter category, the symbol category comprises an undefined and heterogeneous set of exemplars. The symbols lack what Murphy and Medin (1985) refer to as a cohesiveness (viz., there is nothing internal to the set of symbols that associates the individual exemplars with one another). Possibly targets from this type of category are less susceptible to response competition.

A second possibility relates to the contrasting nature of the two categories in the letter-symbol task. In the letter-letter and the letter-digit classification task, it may be argued that the two categories are more comparable in their cohesiveness (e.g., both letters and digits come from well-learned categories). In the letter-symbol task, the letter category, which is composed of a well-defined finite class, is contrasted with a category composed of exemplars from an undefined heterogeneous set. Thus, the letter-symbol task involves classifying two contrasting categories, whereas the letter-letter task contrasts two comparable categories. This difference in contrast between the two categories could influence the classification decision rule, which in turn could have an effect on response competition.

In Experiment 4, subjects classified the familiar and unfamiliar symbols used in the previous experiments. If the contrasting nature of the letter-symbol categories is responsible for the asymmetry in response priming, then it should be possible to restore large and equivalent response-priming effects in both categories by using a symbol-symbol classification task. However, if the absence of response priming is related to the low degree of cohesiveness in the symbol categories, then a symbolsymbol task should result in nonsignificant response priming in both symbol categories.

\section{Method}

The method used in this experiment was identical to that used in Experiment 3, except that the category of familiar letters was replaced by the category of unfamiliar symbols used in Experiments 1 and 2. The subjects were instructed to respond to the center symbol as being either familiar or unfamiliar. Eighteen different students from the pool described previously served as subjects. Data from 2 additional subjects were discarded, 1 for prior knowledge of the unfamiliar symbols and 1 for excessive error rates (20\% or more errors in any one prime condition).

\section{Results and Discussion}

Response priming. The means of subjects' median RTs and the mean percentages of errors are presented in $\mathrm{Ta}$ ble 4 . As in the previous experiments, there was a significant response-priming $\times$ category interaction $[F(1,17)=$ $5.85, p<.05]$, reflecting a larger response-priming effect for the familiar symbol category than for the unfamiliar symbol category. An analysis of simple effects showed that there was a significant response-priming effect for both the familiar symbols $[F(1,17)=170.6$, $p<.001]$ and the unfamiliar symbols $[F(1,17)=49.1$, $p<.001]$. The analysis of the error data indicated that response priming and category did not interact $[F(1,17)=$ $1.06, p>.1]$. There were more errors on incompatible trials than on compatible trials for both the familiar symbols $[F(1,17)=7.8, p<.05]$ and the unfamiliar symbols $[F(1,17)=18.4, p<.001]$.

These results suggest that the contrasting nature of the categories used in the classification task is, at least, partially responsible for 'the asymmetry effect. In Experi-' ments 1-3, where the letters were contrasted with either the familiar or the unfamiliar symbols, there was asymmetry with no evidence of response competition for the symbol targets. When the two sets of symbols were contrasted, we continued to find asymmetric response priming, but there was evidence of a substantial responsepriming effect for both categories. The idea that response competition is influenced, in some manner, by the contrast in the cohesiveness between the letter and the symbol categories suggests that the classification decision rule (e.g., representing the problem as letter versus nonletters) may influence response competition. Our finding that the unfamiliar symbols showed less response priming than the familiar symbols did suggests that the two symbol categories were not entirely comparable. Possibly the familiar symbol category was more cohesive.

Stimulus priming. There was a significant stimuluspriming effect for both the familiar symbols $[F(1,17)=$ $20.1, p<.001]$ and the unfamiliar symbols $[F(1,17)=$ $17.22, p<.001]$. There was no evidence that stimulus priming interacted with familiarity $(F<1)$. The analysis of the error data indicated that the identical and compatible trials did not differ for either the familiar symbols $[F(1,17)=1.7, p>.1]$ or the unfamiliar symbols

Table 4

Mean Reaction Times (RT)

and Proportions of Errors (E) for Experiment 4, as a Function of Target Familiarity and Type of Prime

\begin{tabular}{lccccc}
\hline & \multicolumn{4}{c}{ Target } \\
\cline { 2 - 3 } \cline { 5 - 6 } \multicolumn{1}{c}{ Type of Prime } & \multicolumn{2}{c}{ Familiar } & & Unfamiliar \\
\cline { 2 - 3 } \cline { 5 - 6 } & RT & E & & RT & E \\
\hline Identical & 458 & 0.4 & & 484 & 0.2 \\
Response-compatible & 479 & 0.7 & & 503 & 0.5 \\
Response-incompatible & 527 & 2.8 & & 538 & 2.0 \\
\hline
\end{tabular}


$[F(1,17)=1.3, p<.1]$. These results are consistent with those of the previous experiments, providing no indication that stimulus priming is related to familiarity.

\section{EXPERIMENT 5}

The results from Experiment 4 suggested that the response-priming asymmetry effect is influenced by the nature of the classification decision rule that is induced by the contrast in the two response classes. This finding did not, however, eliminate the possibility that the intrinsic structure of the categories constituting the response classes might also have an independent effect on response priming. For example, it remained possible that categories low in cohesiveness are less susceptible to response competition, and this effect might operate independently of the response-class contrast effect found in Experiment 4. We investigated this possibility in Experiment 5 by using a mixed-category task (cf. LaBerge, 1981), in which letters and symbols were assigned to both response classes (i.e., half of the stimuli assigned to each response were letters and half were symbols). With the mixed-category classification task, the two response classes were completely comparable, and it was possible to compare response priming for letter and for symbol targets within a response class.

If the asymmetry in response priming was simply the result of the contrasting nature of the two response classes, we expected we would see uniform response-priming effects for both the letter and the symbol targets. However, if we continued to find an asymmetry effect (i.e., less response priming with the symbol targets), it would suggest that the effect was related to some property of the symbol category.

It may be noted that a number of researchers have investigated priming effects using the mixed-category task with letters and digits (Flowers \& Wilcox, 1982; LaBerge, 1981 ; Proctor \& Fober, 1985, 1988). With the excepon of the LaBerge paper, these studies failed to report separate statistical analyses of letter and digit targets. Although LaBerge found some evidence of responsepriming asymmetry, the pattern was inconsistent across the letter and digit targets.

\section{Method}

The general procedures in Experiment 5 were similar to those in Experiment 4, except that each response class was composed of two letters and two familiar symbols. The stimuli $F, H$, \#, and ? were assigned to one response class, and the stimuli $R, S, I$, and \& were assigned to the other response class. This resulted in five types of trials, described in terms of the relationship between the primes and the target. The trial types and the overall proportion of trials on which they occurred were as follows: identical (ID) (e.g., \#\#\#) $12.5 \%$; compatible response-same category (CS) (e.g., ? ??) $18.75 \%$; compatible response-different category (CD) (e.g., F\#F) $18.75 \%$; incompatible response-same category (ICS) (e.g., \&\#\&) $25.0 \%$; and incompatible response-different category (ICD) (e.g., S\#S) $25 \%$. The subjects were given a 128-trial practice session followed by a 448-trial experimental session. A card displaying the correct assignment of the eight stimuli was left available to each subject throughout the experiment. Twenty-four students from the population used in the previous experiments served as subjects. Three subjects were discarded for excessive error rates $(20 \%$ or more errors in any one prime condition).

\section{Results and Discussion}

The means of subjects' median RTs and percentages of errors are presented in Table 5. Before proceeding with the analysis of response latencies, we can briefly dispense with the error data. It can be seen from Table 5 that error rates were in general quite low, varying between mean percentages of .9 and 2.3 across the various prime conditions. An overall analysis of error rates, however, indicated a significant effect of prime type $[F(4,92)=2.93$, $p<.05]$. Neither type of category nor the prime type $\times$ category interaction was significant (both $F \mathrm{~s}<1.0$ ). From an inspection of Table 5 , it can be seen that error rates tended to be somewhat lower in the ID and CS prime conditions.

Response priming. The analysis of response priming involved a comparison of the CS, CD, ICS, and ICD prime conditions. This overall analysis involving these four conditions showed a significant response-priming effect $[F(3,69)=8.33, p<.001]$, no evidence of a category effect $(F<1.0)$, and only marginal evidence of a response-priming $\times$ category interaction $[F(3,69)=2.14$, $p=.103$ ].

The test for response priming in the previous experiments, using the unmixed classification task, involved a comparison between compatible and incompatible prime conditions. For the unmixed classification task, a compatible prime was a member of both the same category and the same response as the target, whereas an incompatible prime belonged to a different category and response. The comparable comparison in the mixed classification task is between the CS and ICD conditions. A contrast analysis involving these two conditions resulted in a significant prime $\times$ category interaction $[F(1,23)=$ $6.05, p<.05]$, reflecting the smaller degree of response priming for the symbol category $(12 \mathrm{msec})$ than for the letter category $(36 \mathrm{msec})$. These response-priming effects were significant for both the symbol $[F(1,23)=4.94$, $p<.05]$ and the letter $[F(1,23)=16.4, p<.001]$ targets.

These findings suggest that the asymmetry effect may be influenced both by the decision rule and by an intrinsic characteristic of the symbol category (e.g., noncohe-

Table 5

Mean Reaction Times (RT) and Percentages of Error Rates (E) for Experiment 5, for Letter and Symbol Categories and Type of Prime

\begin{tabular}{lccccc}
\hline & \multicolumn{4}{c}{ Target } \\
\cline { 2 - 3 } \multicolumn{1}{c}{ Type of Prime } & \multicolumn{2}{c}{ Letter } & & \multicolumn{2}{c}{ Symbol } \\
\cline { 2 - 3 } \cline { 5 - 6 } \cline { 5 - 6 } & $\mathrm{RT}$ & $\mathrm{E}$ & & $\mathrm{RT}$ & $\mathrm{E}$ \\
\hline Identical & 683 & 0.89 & & 637 & 1.64 \\
Compatible-same & 631 & 1.29 & & 642 & 1.49 \\
Compatible-different & 673 & 2.08 & & 660 & 2.28 \\
Incompatible-same & 656 & 2.08 & & 660 & 2.32 \\
Incompatible-different & 667 & 1.49 & & 654 & 1.56 \\
\hline
\end{tabular}


siveness). First, with regard to the decision rule, in the previous letter-symbol experiments (Experiments 1-3), we found that there was a significant response-priming effect only for the letter targets. The fact that we observed significant response priming for symbol targets in both Experiments 4 and 5 suggests that making the response categories more comparable leads to an increase in the response-priming effect for the symbol targets. The comparability of the response categories was increased both by making the stimulus sets constituting the response categories more similar to one another (Experiment 4) and by equating the response categories by using mixed stimulus sets (Experiment 5). Second, the significant asymmetry effect with the mixed-category task, in which the response categories are completely comparable, suggests that the asymmetry effect can not be explained entirely in terms of the contrasting nature of the letter and symbol categories. It appears that some property of the symbol category itself may also play a critical role in producing the asymmetry.

As noted above, all of our previous assessments of response priming involved comparisons between CS and ICD prime conditions. This comparison confounds response and category effects. As a consequence, it cannot be determined whether the smaller response-priming effects found with the symbol targets reflect an ineffectiveness for the letter primes as a source of interference, or whether it is the case that symbol targets are less susceptible to response competition. This ambiguity was resolved by a comparison between the CS and CD prime conditions, which resulted in a marginally significant prime $x$ category interaction effect $[F(1,23)=3.56, p<.10]$. This interaction suggests that incompatible symbol primes produced less interference in the classification of symbol targets $(12 \mathrm{msec})$ than did incompatible letter primes in the classification of letter targets $(36 \mathrm{msec})$. Overall, it appears that there was less interference from an incompatible prime (letter or symbol) when the target was a symbol rather than a letter. These results suggest that symbol targets are less susceptible to response competition than letter targets are.

Stimulus priming. The investigation of stimulus priming in the mixed-category task has received a great deal of attention in the recent literature because of the occurrence of an unexpected identity-suppression effect (Flowers \& Wilcox, 1982; LaBerge, 1981; Proctor \& Fober, 1985, 1988). Using a letter-digit mixed-category task these studies found that, with simultaneous presentation of the prime and target, RTs are slower on ID trials than on CS trials. Analysis of the ID and CS conditions in the present letter-symbol task showed a significant stimulus-priming $\times$ category interaction $[F(1,23)=8.87$, $p<.01]$. From Table 5, it can be seen that there was a 51-msec identity-suppression effect with the letter targets $[F(1,23)=7.84, p>.01]$ and a small $(6-\mathrm{msec})$ nonsignificant facilitation effect for the symbols $(F<1.0)$.

The absence of an identity-suppression effect for the symbol targets again suggests that the symbols and let- ters are processed differently. Because the present study involved a 100 -msec prime-target SOA, as opposed to the simultaneous presentation used in the previously mentioned studies, the results may have only limited implications for understanding the identity-suppression effect. However, it does appear that symbols are less susceptible to identity suppression than the letters are. Proctor and Fober (1988) showed that the identity-suppression effect with letters and digits is eliminated when subjects are given extensive practice. This finding leads them to conclude that identity suppression must be related to the increased response-selection demands that are present in the mixed-category task. By this account, the absence of suppression for the symbol targets in the present results implies that response selection was more efficient for the symbols than the letters.

\section{GENERAL DISCUSSION}

It has been shown repeatedly that classification RTs are slowed by the presence of an incompatible prime (Taylor, 1977). This inhibition is assumed to result from competing response tendencies emanating from the incompatible prime stimuli that are processed to the point of incipient response activation (cf. Eriksen \& Schultz, 1979). Some direct support for the existence of response competition has been provided in recent studies, which have demonstrated that incompatible primes generate event-related brain potentials and electromyographic activity associated with the competing response (Coles et al., 1985; Gratton, Coles, Sirevaag, Eriksen, \& Donchin, 1988). Consistent with this conceptualization of response competition, the interference disappears when a disjunctive classification task is used (Grice, Canham, \& Schafer, 1982). Apparently both classes of stimuli must be associated with different overt responses.

The general picture that emerges from this literature is that response competition is a robust and pervasive phenomenon. Given the presentation of an incompatible prime in the immediate temporal and spatial context of the target stimulus, we can expect to observe a reliable response-competition effect. Nothing in this model of response competition suggests that the above generalization should be qualified depending on the nature of the stimuli or the categories that are being classified. From this perspective, Shapiro and Krueger's (1983) failure to find response competition with the distorted letter targets was completely unexpected.

The primary aim in this study was to investigate the conditions surrounding the occurrence of the asymmetrical response-priming effect reported by Shapiro and Krueger (1983). Our results (Experiments 1-3) corroborate Shapiro and Krueger's, showing that an incompatible letter prime failed to slow the speeded classification of a symbol target, whereas a symbol prime significantly slowed response latencies to a letter target. This asymmetry in response priming was found across variations in the prime-target SOA and manipulations in the familiarity of the stimuli 
constituting the symbol category. Given that the asymmetry effect is highly reliable, it poses a serious problem for our assumptions regarding the operation of response competition.

One possible approach to explaining the asymmetry effect is to assume that response priming involves a significant facilitory component and to then argue that letter primes facilitate responses to letter targets more than symbol primes facilitate responses to symbol targets. In other words, there is a larger category-priming effect associated with compatible letters than with compatible symbols.

This differential facilitation account of the asymmetry effect runs into two problems. First, when a neutral prime condition was included in the design of Experiment 2, there was no support for differential facilitation. The facilitation from compatible primes for letter targets $(9 \mathrm{msec})$ was virtually identical to what was found with compatible primes for symbol targets $(11 \mathrm{msec})$. It may be the case that the so-called no-noise neutral used in Experiment 2 was "fast"' on some absolute basis, but the absolute magnitude of these effects is not at issue. Finding that the difference in response latencies between the compatible and neutral conditions was the same for the letter and symbol categories indicates that the asymmetry in response priming can not be accounted for in terms of differential category facilitation from compatible primes.

A second problem for the differential facilitation account arises when there is a nonsignificant responsepriming effect, as was found with the symbol targets in Experiments 1-3. It can be argued that this occurs because there is no facilitation from compatible symbol primes, but this implies that there is no response competition from letter primes. This assumption is made without justification and is in direct contradiction with all the evidence in the literature for response competition (e.g., Coles et al., 1985; Eriksen \& Schultz, 1979; Gratton et al., 1988; Grice \& Gwynne, 1985; Taylor, 1977).

Assuming that the asymmetry effect cannot be accounted for simply in terms of differential facilitation, we now turn our attention to the conditions that appear to be responsible for the asymmetry in response competition. The results from Experiment 1 indicated the effect was not sensitive to manipulations in the prime-target SOA. This is important if it is assumed that symbols are processed at a different rate than letters are. For example, if letters are encoded more rapidly than symbols, it may be argued that subjects inhibit the response to the incompatible letter primes rapidly enough so that they do not slow the classification of the symbol target. The absence of a significant increase in response priming for the symbol targets as the SOA decreased to 0 msec poses a problem for time-course explanations of the above type.

In Experiment 3, the unfamiliar symbols were replaced with highly familiar symbols. This rather dramatic change in the familiarity of the exemplars constituting the symbol category had no discernible effect on the results. Although there were numerous other properties of the stimuli that we might have investigated (e.g., verbal encodability), it was our impression at the time that the asymmetry effect was related to the decision rule. With both the familiar and the unfamiliar symbols, subjects often commented that they classified the stimuli as letters versus nonletters. This representation of the task was thought to be related to the contrast in the cohesiveness (Murphy \& Medin, 1985) between the letter and symbol categories. In studies resulting in symmetrical responsecompetition effects, the classification task has always involved two categories that appeared to be relatively comparable in cohesiveness (e.g., letters versus letters, Eriksen \& Schultz, 1979; or letters versus digits, Johnson, Boroughs, Weisgerber, \& Santos-Gomez, 1986).

When we made the two response categories more comparable by contrasting the familiar and unfamiliar symbol categories (Experiment 4), both symbol categories showed significant response priming, whereas neither showed response priming when contrasted with the letter category. This suggests that response competition is related to the classification decision rule. The symbolsymbol task also resulted in an asymmetry effect with greater response priming associated with the more familiar symbols. This may simply indicate that the familiar and unfamiliar symbol categories are not entirely comparable in cohesiveness.

We made the two response classes more comparable in Experiment 5 by using a mixed letter-symbol category task. In the mixed-category task, both response categories are equally noncohesive, being a mix of letters and symbols, and in this sense the classes are as comparable as a letter-letter classification task. On this basis, we expected to find symmetrical and significant responsepriming effects for both the letters and the symbols with the mixed-category task. The results, however, continued to show a significant response-priming asymmetry. Again, there was less response priming with the symbol targets than with the letter targets. These results indicate that the asymmetry effect is not completely tied to the decision rule. Rather, it appears that the effect is also related to some property of the symbol categories.

When we found the asymmetry effect using the unmixed-category task, it could not be determined if the smaller response-priming effect with the symbol targets occurred because the incompatible letter primes were a less potent source of interference, or if the symbol targets were simply less susceptible to inhibition. The results from the mixed-category task indicated that there was less response priming for symbol targets than for letter targets, for both letter and symbol incompatible primes. These results suggest that a symbol target is less susceptible to response competition than a letter target is. This, of course, does not tell us why symbol categories are less susceptible to interference, but it does narrow the search for an explanation. For example, it has been found that interference is related to the categorical nature of the noise (Eriksen \& O'Hara, 1982). This finding would be relevant 
to the asymmetry effect, had we not found that differential levels of inhibition were more related to the nature of the target stimulus than to the nature of the primes.

In conclusion, with respect to response-priming asymmetry, it was our goal to first establish the reliability of the finding and then to define some of the conditions under which the effect is observed. With regard to the first goal, the present experiments have clearly established the reliability of the asymmetry effect. Toward the second goal, the findings suggest that both the classification decision rule and the cohesiveness of the category are related to the asymmetry. If it is agreed that a significant component of this asymmetry is related to differential inhibition (i.e., not exclusively differential facilitation), the results suggest that response competition does not operate in the simple, direct, and ubiquitous manner we had previously assumed.

Whereas the present findings contain some clear implications regarding the conditions surrounding the $o c$ currence of response competition, they also leave us with the perplexing question of how category cohesiveness and the decision rule influence response competition. There is a considerable amount of research to be completed before we have a complete understanding of response competition. We have speculated that a critical property of the symbol category was its low cohesiveness, and that category comparability was influencing the decision rule. With a clearer understanding of the factors controlling the operation of response competition, we can hopefully gain a better understanding of the causal mechanisms underlying these effects.

We now turn our attention to the stimulus-priming effects. A secondary aim of the present study was to investigate the effect of character familiarity on stimulus priming. Shapiro and Krueger (1983) had previously reported finding no evidence of priming from an identical prime when the stimuli were distorted letters. They attributed this finding to the distorted letters' being unfamiliar and therefore not having a well-formed pathway that would allow for code-activation effects (Posner \& Snyder, 1975). Shapiro and Krueger's (1983) findings, however, are in sharp contrast with the present results, where we provide convincing evidence that the classification of an unfamiliar stimulus is facilitated when it is preceded by identical primes. One distinction between the two studies that might account for the discrepant results is that the unfamiliar stimuli in Shapiro and Krueger's study differed from the familiar only in terms of the addition (Experiment 1) or deletion (Experiment 2) of a single line segment, whereas in the present study familiar and unfamiliar characters differed in several features. Thus, it is possible that the subjects in the Shapiro and Krueger study used a more analytically based decision rule (e.g., based on the presence or absence of a single feature) and focused attention to detect a specific feature. Although we know of no direct evidence indicating that decision strategies would reduce identity priming, this remains the most obvious difference between the two studies.

The observation of stimulus priming with the unfamiliar characters in the present study is compatible with models in which priming effects are based on an episodic trace of the prime rather than on the activation of a permanent memory code (cf. Feustel, Shiffren, \& Salasoo, 1983; Jacoby \& Brooks, 1984). However, it is still possible for code-activation types of models (Morton, 1969, 1979; Posner, 1978) to explain the current results by assuming that memory representations of the unfamiliar characters were formed early in the experimental session, through repeated exposure. Along these lines, Salasoo, Shiffren, and Feustel (1985) have presented evidence that the formation of memory codes may be a rapid process requiring relatively few exposures.

\section{REFERENCES}

Coles, M. G. H., Grattón, G., Bashore, T. R., Eriksen, C. W., \& Donchin, E. (1985). A psychophysiological investigation of the continuous flow model of human information processing. Joumal of Experimental Psychology: Human Perception \& Performance, 11, 529-553.

ERIKSEN, B. A., \& ERIKSEN, C. W. (1974). Effects of noise letters upon the identification of a target letter in a nonsearch task. Perception \& Psychophysics, 16, 143-149.

ERIKSEN, C. W., \& O'HARA, W. P. (1982). Are nominal same-different matches slower due to differences in level of processing or to response competition? Perception \& Psychophysics, 32, 335-344.

ErJKsen, C. W., SChultz, D. W. (1979). Information processing in visual search: A continuous flow conception and experimental results. Perception \& Psychophysics, 25, 249-263.

Feustel, T. C., Shiffren, R. M., Salasoo, A. (1983). Episodic and lexical contributions to the repetition effect in word identification. Journal of Experimental Psychology: General, 112, 309-346.

FLowers, J. H., WiLcox, N. (1982). The effect of flanking context on visual classification: The joint contribution of interactions at different processing levels. Perception \& Psychophysics, 32, 581-591.

Gratton, G., Coles, M. G. H., Sireva aG, E. J., Eriksen, C. W., Donchin, E. (1988). Pre- and poststimulus activation of response channels: A psychophysical analysis. Journal of Experimental Psychology: Human Perception \& Performance, 14, 331-344.

Grice, G. R., Boroughs, J. M., Canham, L. (1984). Temporal dynamics of associative interference and facilitation produced by visual context. Perception \& Psychophysics, 36, 499-507.

Grice, G. R., Canham, L., \& SChafer, C. (1982). Role of the response in associative interference. Bulletin of the Psychonomic Society, 20, 214-216.

Grice, G. R., \& GWYNNE, J. W. (1985). Temporal characteristics of noise conditions producing facilitation and interference. Perception \& Psychophysics, 37, 495-501.

JACOBY, L. L., \& BROOKS, L. R. (1984). Nonanalytic cognition: Memory, perception, and concept learning. In G. H. Bower (Ed.), The psychology of learning and motivation (Vol. 18, pp. 1-47). New York: Academic Press.

Johnson, P. J., Boroughs, J. M., Weisgerber, S. A., \& SantosGoMEz, L. (1986, November). Familiarity and congruence effects on stimulus priming. Paper presented at the meeting of the Psychonomic Society, New Orleans, LA.

JoNides, J., \& MACK, R. (1984). On the cost and benefit of cost and benefit. Psychological Bulletin, 96, 29-44.

KIRSNER, K. \& DUNN, J. (1985). The perceptual record: A common factor in repetition priming and attribute retention? In M. I. Posner 
\& D. S. M. Marin (Eds.), Attention and performance XI (pp. 547565). Hillsdale, NJ: Erlbaum.

LABERGE, D. (1981). Unitization and automaticity in perception. In J. H. Flowers (Ed.), Nebraska Symposium on Motivation: Cognitive processes (Vol. 28, pp. 53-71). Lincoln, NB: University of Nebraska Press.

Miller, J. (1987). Priming is not necessary for selective-attention failures: Semantic effects of unattended, unprimed letters. Perception \& Psychophysics, 41, 419-434.

Morton, J. (1969). Interaction of information in word recognition. Psychological Review, 76, 165-178.

MoRTON, J. (1979). Facilitation in word recognition: Experiments causing a change in the logogen model. In P. A. Kolers, M. E. Wrolstal, \& H. Bonma (Eds.), Processing of visible language (Vol. 1, pp. 259. 268). New York: Plenum.

MuRPhy, G. L., MEDIN, D. L. (1985). The role of theories in conceptual coherence. Psychological Review, 92, 289-316.

Posner, M. I. (1978). Chronometric explorations of mind. New York: Halsted.

Posner, M. I., \& SNyder, C. R. R. (1975). Attention and cognitive control. In $\mathrm{R}$. Solso (Ed.), Information processing and cognition: The Loyola Symposium. Potomac, MD: Erlbaum.
Proctor, R. W., \& Fober, G. W. (1985). Repeated-stimulus superiority and inferiority effects in the identification of letters and digits. Perception \& Psychophysics, 38, 125-134.

Proctor, R. W., \& Fober, G. W. (1988). A response-selection basis for the mixed-category, repeated-stimulus inferiority effect. Perception \& Psychophysics, 44, 182-190.

Salasoo, A., Shiffren, R. M., Feustel, T. C. (1985). Building permanent memory codes: Codification and repetition effects in word identification. Journal of Experimental Psychology: General, 114, 50-77.

ShaFFer, W. O., \& LABERGE, D. (1979). Automatic semantic processing of unattended words. Joumal of Verbal Learning \& Verbal Behavior, $18,413-426$.

ShaPIRo, R. G., \& Krueger, L. E. (1983). Effect of similarity of surround on target-letter processing. Journal of Experimental Psychology: Human Perception \& Performance, 9, 547-559.

TAYLOR, D. A. (1977). Time course of context effects. Journal of Experimental Psychology: General, 106, 404-426.

(Manuscript received April 29, 1988; revision accepted for publication June 20,1989 .) 